\title{
Serum sE-Selectin Levels and Carcinoembryonic Antigen mRNA-Expressing Cells in Peripheral Blood as Prognostic Factors in Colorectal Cancer Patients
}

\author{
Patrizia Ferroni, MD, $\mathrm{PhD}^{1}$; Mario Roselli, MD²,3; Antonella Spila, $\mathrm{PhD}^{1}$; Roberta D’Alessandro, $\mathrm{PhD}^{1}$; \\ Ilaria Portarena, $\mathrm{MD}^{3}$; Sabrina Mariotti, MD³; Raffaele Palmirotta, MD, PhD'; Oreste Buonomo, $\mathrm{MD}^{4}$; \\ Giuseppe Petrella, $\mathrm{MD}^{4}$; and Fiorella Guadagni, MD, $\mathrm{PhD}^{1}$
}

BACKGROUND: This study analyzed the possible prognostic value of presurgical serum soluble (s)E-selectin levels and/or carcinoembryonic antigen (CEA) mRNA positivity in predicting the disease-free survival of colorectal cancer (CRC) patients. METHODS: CEA mRNA (obtained from blood-borne cells by reverse transcriptase-polymerase chain reaction $[R T-P C R]$ ), tumor necrosis factor- $\alpha$ (TNF- $\alpha$ ), and SE-selectin levels were analyzed in blood samples obtained from 78 patients with primary $(n=62)$ or recurrent $(n=16)$ CRC, 40 patients with benign colorectal $(C R)$ diseases, and 78 controls. RESULTS: CEA mRNA positivity by RT-PCR was significantly associated with advanced stage ( $P$ $<$.05). Median baseline sE-selectin levels were higher in patients with CRC (43 ng/mL) compared with controls (36 ng/mL) or patients with benign CR diseases $(31 \mathrm{ng} / \mathrm{mL}, P<.001$ ). These were significantly associated with $C E A$ mRNA positivity by RT-PCR $(P<.05)$. Multivariate analysis by forward stepping showed that elevated TNF- $\alpha(P=$ .001) and CEA mRNA positivity by RT-PCR $(P=.0001)$ were independent predictors of elevated baseline sE-selectin levels. Positive presurgical sE-selectin levels were associated with an increased recurrence rate compared with patients with low levels of this molecule $(P<.001)$. Positivity for both CEA mRNA and sE-selectin had a negative prognostic impact, with a 5-year recurrence-free survival rate of 51\% compared with $95 \%$ of patients with negative parameters $(P<.05)$. CONCLUSIONS: Detection of presurgical serum sE-selectin levels and CEA mRNA-positive blood-borne cells in CRC patients might provide useful prognostic information in terms of recurrence-free survival, either alone or in combination, and may help in the choice of more aggressive treatment and/or more strict follow-up procedures in high-risk patients. Cancer 2010;116:2913-21. (c) 2010 American Cancer Society.

KEYWORDS: colorectal cancer, blood-borne cells, carcinoembryonic antigen, sE-selectin, metastasis.

Several lines of evidence suggest that the organ selectivity of colorectal cancer (CRC) cells for the liver involves the binding of the metastatic cells to endothelial E-selectin. ${ }^{1,2}$ Tumor cell entry into the hepatic microvasculature can trigger a rapid, proinflammatory cascade that begins with increased local tumor necrosis factor- $\alpha$ (TNF- $\alpha$ ) production by activated Kupffer cells and leads to up-regulated E-selectin expression on the hepatic sinusoidal vessels, ${ }^{3,4}$ which serve not only for initial adhesion and rolling, but also for subsequent diapedesis into metastatic sites. ${ }^{2}$ The involvement of TNF- $\alpha$ and E-selectin expression in the metastatic cascade has been also proposed by Sturm et al, who suggested that TNF- $\alpha$ facilitates tumor cell adhesion and extravasation by inducing the expression of E-selectin and other adhesion molecules in the liver vasculature of syngeneic mice. ${ }^{5}$

Corresponding author: Patrizia Ferroni, MD, PhD, Department of Laboratory Medicine and Advanced Biotechnologies, IRCCS San Raffaele, Via della Pisana 235, 00163, Rome, Italy; Fax: (011) 39 (06) 66130407; Patrizia.ferroni@sanraffaele.it

${ }^{1}$ Department of Laboratory Medicine and Advanced Biotechnologies, Scientific Institute for Research, Hospitalization and Health Care (IRCCS) San Raffaele, Rome, Italy; ${ }^{2}$ Scientific Institute for Research, Hospitalization and Health Care (IRCCS) San Raffaele, Rome, Italy; ${ }^{3}$ Division of Medical Oncology, Department of Internal Medicine, University of Rome "Tor Vergata," Tor Vergata Clinical Center, Rome, Italy; ${ }^{4}$ Department of Surgery, University of Rome "Tor Vergata," Tor Vergata Clinical Center, Rome, Italy

We thank Barbara Leone for her expert technical assistance.

DOI: 10.1002/cncr.25094, Received: August 14, 2009; Revised: September 28, 2009; Accepted: October 7, 2009, Published online March 24, 2010 in Wiley InterScience (www.interscience.wiley.com) 
It is currently recognized that carcinoembryonic antigen (CEA) is capable of activating hepatic macrophages or Kupffer cells via binding to a CEA receptor. ${ }^{6}$ Moreover, the demonstration that CEA itself functions as an alternative receptor for E-selectin to mediate CRC cell adhesion has led to the formulation of a hypothesis concerning the apparent enhanced metastatic potential associated with CEA overexpression on CRC cells and the critical role of selectins in metastatic spread. ${ }^{7}$ Accordingly, elevated soluble (s)E-selectin and TNF- $\alpha$ levels have been found in sera from CRC patients, and both TNF- $\alpha$ and serum CEA levels have been independently associated with increased sE-selectin levels. ${ }^{8}$

One of the newer areas being explored in the management of cancer is the use of reverse transcriptase-polymerase chain reaction (RT-PCR) to analyze the blood of cancer patients for the detection of mRNA expressed in tumor cells. ${ }^{9,10}$ The CEA gene is 1 of the most widely expressed genes in cancer cells, and serum CEA protein is currently used to follow the course of therapy in the management of colorectal carcinoma. ${ }^{11}$ Nonetheless, it has been shown that only approximately half of CRCs shed CEA in levels sufficient for their detection in monitoring therapy. ${ }^{12}$ Numerous studies have reported the use of RTPCR technology to detect CEA message in tumor cells from peripheral blood of cancer patients, ${ }^{13}$ suggesting that longitudinal analyses of blood-borne cells expressing CEA by RT-PCR may be useful for the clinical management of CRC and other CEA-expressing tumors in terms of prognosis or in the analysis of the response to therapy either alone ${ }^{14-17}$ or in combination with other molecular markers. ${ }^{18,19}$

In light of these considerations, a follow-up study of CRC patients was performed to investigate the possible association of presurgical serum sE-selectin levels with the positivity for CEA mRNA by RT-PCR in peripheral blood cells, as well as their prognostic value in predicting recurrence-free survival.

\section{MATERIALS AND METHODS \\ Patients}

Seventy-eight consecutive patients with CRC ( 40 men, 38 women; mean age, $58 \pm 12$ years), treated at the Tor Vergata Clinical Center, entered the study. Patients were histologically diagnosed with primary $(n=62)$ or relapsing (metastasis to the liver, $\mathrm{n}=8$; peritoneum, $\mathrm{n}=4$; lung, $\mathrm{n}=2$; and multiple metastasis, $\mathrm{n}=2$ ) colorectal adenocarcinoma. Primary CRC was pathologically staged according to the International Union Against Cancer TNM Classification: stage I $(n=6)$, stage II $(n=35)$, stage III $(\mathrm{n}=19)$, and stage IV ( $\mathrm{n}=2$, with a single resectable liver metastasis). All patients were observed from the time of diagnosis of primary tumor for at least 3 years after surgery or until disease recurrence.

As control groups, 78 healthy age- and sex-matched subjects (mean age, $57 \pm 13$ years) and 40 subjects with benign colorectal diseases (21 men, 19 women; mean age, $53 \pm 13$ years $)$ including polyps $(n=28)$ and chronic ulcerative colitis in stable phase of disease $(\mathrm{n}=$ 12) were also evaluated. The study was performed with the appropriate institutional ethics approval and in accordance with the principles embodied in the Declaration of Helsinki, and informed consent was obtained from each subject.

\section{Sample Collection and Processing}

Blood samples from primary CRC patients were drawn within 1 week before surgery, or before neoadjuvant chemotherapy and/or irradiation. Samples from patients with metastatic disease were obtained at the time of clinical diagnosis, and before any treatment. Samples from patients with benign disease were drawn at the time of endoscopy. Postsurgical blood samples were obtained from 33 primary CRC patients (stage I, $\mathrm{n}=2$; stage II, $\mathrm{n}=18$; stage III, $\mathrm{n}=11$; and stage IV, $\mathrm{n}=2$ ) who provided informed consent to withdraw additional blood samples during follow-up.

After an overnight fast and a rest of at least 20 minutes, blood was drawn from each consenting subject at the middle of vein puncture (to avoid contamination with epidermal epithelial cells) into 7-mL Vacutainers containing no additive or ethylenediaminetetraacetic acid (EDTA). Serum samples were aliquoted, coded, and stored at $-40^{\circ} \mathrm{C}$ until the assays were performed.

Peripheral blood mononuclear cells (PBMCs) were isolated from EDTA blood, after dilution in phosphatebuffered saline (PBS), by density gradient centrifugation using Lympholyte-H (CELBIO S.p.A., Siziano, Italy) at $1500 \mathrm{~g}$ for 35 minutes. The interface cells were removed, washed in sterile PBS $1 \times$, pelleted, and resuspended in $2 \mathrm{~mL}$ of PBS. The cells were then counted and pelleted again at $2500 \mathrm{~g}$ for 15 minutes. The cell pellets were dissolved in a denaturing solution containing guanidinium thiocyanate $4 \mathrm{M}$, Na citrate $25 \mathrm{mM}$, Sarcosyl NL 30 $0.5 \%$, and $\beta$-mercaptoethanol $0.1 \mathrm{M}$ and stored at $-80^{\circ} \mathrm{C}$ until RNA extraction. 


\section{RNA Extraction and RT-PCR}

Total RNA was extracted from PBMCs using QIAampRNA Blood Mini Kit (QIAGEN GmbH, Hilden, Germany) according to the manufacturer's instructions. All RNA preparation and handling steps took place in a laminar flow hood, under RNAse-free conditions. The isolated RNA was resuspended in RNAsefree water and stored at $-80^{\circ} \mathrm{C}$ until assay. The concentration, purity, and amount of total RNA were determined using a DU 530 Life Science UV/Vis Spectrophotometer (Beckman Coulter, Fullerton, Calif). RNA prepared from CEA-producing tumor cell line LS174T was used as positive control. Total RNA was preincubated for 15 minutes at $65^{\circ} \mathrm{C}$ with DNase (deoxyribonuclease I, amplification grade, Invitrogen Corporation, Carlsbad, Calif) and RNase inhibitor (RNaseOUT, Invitrogen Corporation). After chilling on ice, reversetranscription of RNA was carried out with the SuperScript III First-Strand Synthesis SuperMix (Invitrogen Corporation), and cDNA was synthesized with 1 to $5 \mu \mathrm{g}$ of total RNA, $50 \mu \mathrm{M}$ of oligodT, and Moloney murine leukemia virus reverse transcriptase, according to the manufacturer's instructions. To obtain specific amplification of reverse-transcribed mRNA, cDNAs were amplified using primer pairs for CEA directed to sequences located in 2 different exons. RNA integrity and the fidelity of cDNA synthesis were verified by PCR amplification of the glyceraldehyde phosphate dehydrogenase (GAPDH) housekeeping gene.

CEA and GAPDH primers were designed according to published Ensembl sequence (CEA Ensembl Gene ID ENSG00000105388; GAPDH Ensembl Gene ID ENSG00000111640), using the Universal ProbeLibrary Assay Design Software (https://www.roche-applied-science. com/sis/rtpcr/upl/adc.jsp).

These primers were as follows: CEA forward primer, 5'-ACCACAGTCACGACGATCAC-3'; CEA reverse primer, 5'-GGAGTTGTTGCTGGTGATGA-3'; GAP DH forward primer, 5'-TCCACTGGCGTCTTCACC$3^{\prime}$; and GAPDH reverse primer, 5'-GGCAGAGATGAT GACCCTTTT-3'.

PCR was performed in a GeneAmp PCR System 9700 (Applied Biosystems, Foster City, Calif). The reaction mixture had a total final volume of $50 \mu \mathrm{L}$ and contained: $50 \mathrm{ng}$ of cDNA, $0.5 \mu \mathrm{M}$ of each oligonucleotide primer, a variable amount of distilled water, and $25 \mu \mathrm{L}$ of HotStarTaq Master Mix (HotStarTaq Master Mix Kit, QIAGEN). PCR was completed after 35 cycles of $30 \mathrm{sec}-$ onds denaturation at $94^{\circ} \mathrm{C}, 30$ seconds annealing at $60^{\circ} \mathrm{C}$, and 1 minute extension at $72^{\circ} \mathrm{C}$. An initial denaturation step of 15 minutes at $95^{\circ} \mathrm{C}$ and a final extension of 10 minutes at $72^{\circ} \mathrm{C}$ were used.

CEA and GAPDH PCR products, respectively of 210 and $114 \mathrm{bp}$, were resolved for each patient's sample and cell lines by electrophoresis on 1\% agarose in Trisacetate-EDTA buffer and observed by ethidium bromide staining of the gel. Signals in each CEA transcript were normalized by the corresponding GAPDH transcript and categorized as having negative, moderate $(+/++)$, or strong $(+++)$ intensity using a Bio-Rad molecular imager system (Quantity One 4.6 software, Hercules, Calif).

\section{Immunoassays}

Serum CEA and CA 19-9 determination was performed using 2-step chemiluminescent microparticle immunoassays on an ARCHITECT i2000 System (Abbott Labs, Chicago, Ill). Serum CA 72-4 levels were measured using a 2-step chemiluminescent microparticle immunoassay on an Elecsys 2010 System (Roche Diagnostics GmBH, Mannheim, Germany). The cutoff limits chosen for sample evaluation were $5 \mathrm{ng} / \mathrm{mL}, 37 \mathrm{U} / \mathrm{mL}$, and $6 \mathrm{U} / \mathrm{mL}$ for CEA, CA 19-9, and CA 72-4, respectively. Serum TNF- $\alpha$ and sE-selectin levels were measured by enzyme-immunometric assays (R\&D Systems, Minneapolis, Minn) according to the manufacturers' instructions. Cutoff limits were calculated on the basis of the 95th percentile of the values observed in healthy subjects and were set at 20 $\mathrm{pg} / \mathrm{mL}$ and $70 \mathrm{ng} / \mathrm{mL}$, respectively. Measurements were done blinded. All samples were assayed in duplicate, and those showing values above the standard curve were retested with appropriate dilutions.

\section{Statistical Analysis}

Data are generally presented as percentage of positive patients. Unless otherwise specified, antigen levels are expressed as median and interquartile range (IQR). Statistical analysis was performed by Kruskal-Wallis test followed by Mann-Whitney $U$ test for post hoc comparison. Differences between percentages were assessed by crosstabulation tables and chi-square analysis. Univariate and multivariate regression analyses were performed to assess the possible associations between variables. Recurrencefree survival was calculated by the Kaplan-Meier method, and the significance level was assessed according to the Cox-Mantel test. The time to the endpoint was calculated from the date of surgery until the event date, with the event being any recurrence, locoregional or systemic, or 
the latest day of follow-up. A sample size of 62 patients participated in the follow-up study, with a $90 \%$ power to detect a 50\% difference in survival rates between patients with either positive or negative variables. Only $P$ values $<.05$ were regarded as statistically significant. All calculations were made using a computer software package (Statistica 8, StatSoft Inc., Tulsa, Okla).

\section{RESULTS}

CEA mRNA from blood cells of patients with different CRC stages was analyzed by RT-PCR before surgery, or before neoadjuvant chemotherapy and/or irradiation. Fifty-one $(65 \%)$ of 78 patients with different stages of

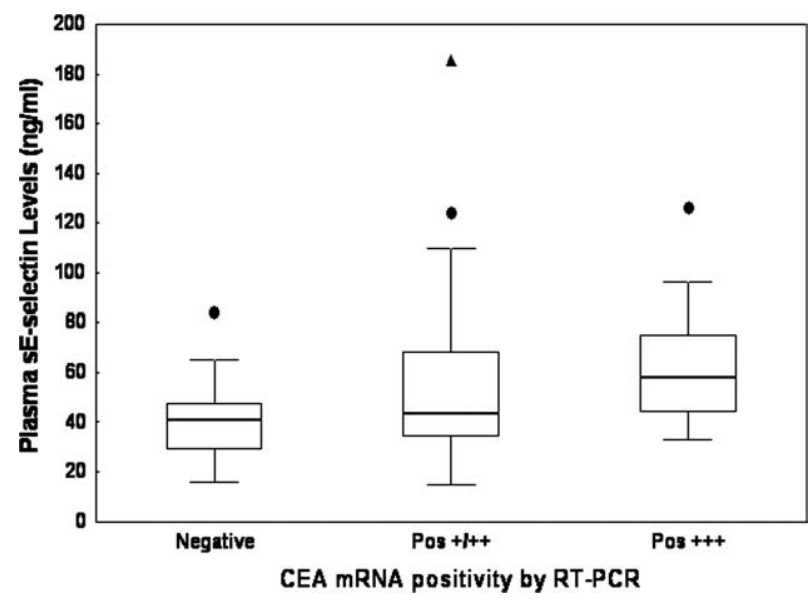

Figure 1. Box plot analysis of sE-selectin levels in colorectal cancer patients stratified on the basis of negative, moderate $(+/++)$, or strong $(+++)$ carcinoembryonic antigen (CEA) mRNA positivity (pos) by reverse transcriptase-polymerase chain reaction (RT-PCR) is shown. Data are presented as median values (solid lines), interquartile range (columns), and nonoutlier (whiskers) ranges. Closed circles indicate outliers; closed triangles, extreme. Kruskal-Wallis analysis of variance and median test: $H=7.48 ; P<.03$. colorectal cancer had blood cells that were positive for CEA mRNA by RT-PCR, whereas none of 40 patients with benign colorectal diseases was positive; 2 of 78 apparently healthy individuals were marginally positive (data not shown). A significant overall association was found between tumor stage and RT-PCR analysis for CEA in the blood of CRC patients, with 54\% CEA mRNA positivity by RT-PCR in patients with early stages compared with $78 \%$ in patients with advanced stages (chi-square, 5.25; $P=.022$ ). Conversely, no significant association was found between CEA mRNA positivity by RT-PCR and serum CEA levels (Mann Whitney test, $P=.159$ ). CEA mRNA positivity was found in $37(63 \%)$ of 59 patients with CEA-negative serum, compared with 14 (74\%) of 19 patients with CEA-positive serum (chisquare, $0.76 ; P=.382$ ).

Median baseline sE-selectin levels were higher in patients with CRC (median [IQR]: 43 [34-62] ng/mL) compared with controls (36 [25-46] ng/mL) or patients with benign colorectal diseases (31 [25-46] ng/mL, $H=$ $17.1, P=.0002)$. Increasing levels of baseline $s E$-selectin were significantly associated with CEA mRNA positivity by RT-PCR $(H=7.48, P=.024)$ (Fig. 1). In particular, only $1(4 \%)$ metastatic patient negative for CEA mRNA before surgery was positive for sE-selectin at baseline $(84.2 \mathrm{ng} / \mathrm{mL})$, compared with $29 \%$ of CEA mRNA-positive patients (chi-square, 7.16; $P=.007$ ). To assess the possible determinants of baseline sE-selectin levels among the clinical and laboratory features of CRC, a multivariate regression analysis was performed in which adjustments were made for the following variables: age, sex, site of primary tumor, grade, stage of disease, serum CEA, CA 19-9 and CA $72-4$ tumor markers, CEA mRNA, and TNF- $\alpha$ levels. The final model by forward stepping showed that elevated TNF- $\alpha$ (regression coefficient, $0.371 ; P=$

Table 1. Multivariate Regression Analysis of Baseline sE-Selectin Levels in Colorectal Cancer Patients

\begin{tabular}{lrrlr} 
Predictor Variable & \multicolumn{2}{c}{ Univariate Analysis } & \multicolumn{2}{c}{ Multivariate Analysis } \\
& $\boldsymbol{\beta}$-Coefficient (SE) & $\boldsymbol{P}$ & $\boldsymbol{\beta}$-Coefficient (SE) & $\boldsymbol{P}$ \\
Sex & $0.048(0.118)$ & .688 & Out \\
Age & $-0.126(0.125)$ & .315 & Out \\
Stage of disease & $-0.002(0.167)$ & .992 & Out \\
Site of primary tumor & $-0.142(0.139)$ & .314 & Out \\
Grade & $0.052(0.145)$ & .723 & Out \\
CEA mRNA & $0.428(0.125)$ & .001 & $0.449(0.110)$ & .0001 \\
Serum CEA levels & $0.051(0.151)$ & .736 & Out \\
Serum CA 19-9 levels & $0.055(0.158)$ & .726 & Out & \\
Serum CA 72-4 levels & $-0.052(0.145)$ & .721 & Out & \\
Serum TNF- $\alpha$ levels & $0.322(0.128)$ & .015 & $0.371(0.110)$ & .0014 \\
\hline SE indicates standard error: CEA & & & &
\end{tabular}

SE indicates standard error; CEA, carcinoembryonic antigen; TNF- $\alpha$, tumor necrosis factor- $\alpha$. 
.0014) and positivity by RT-PCR (regression coefficient, 0.449 ; $P=.0001$ ) were independent predictors of elevated levels of sE-selectin (Table 1).

The interactions among baseline sE-selectin, TNF- $\alpha$ levels, and CEA mRNA positivity by RT-PCR were further analyzed by 2-way analysis of variance. As shown in Figure 2, increased baseline levels of sE-selectin were significantly associated to CEA mRNA positivity in patients with elevated TNF- $\alpha$ levels $(\mathrm{F}=4.68, P=.012$ ). Post hoc analysis by Bonferroni test showed that median pre-

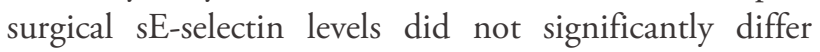
among patients with negative TNF- $\alpha$ levels and either

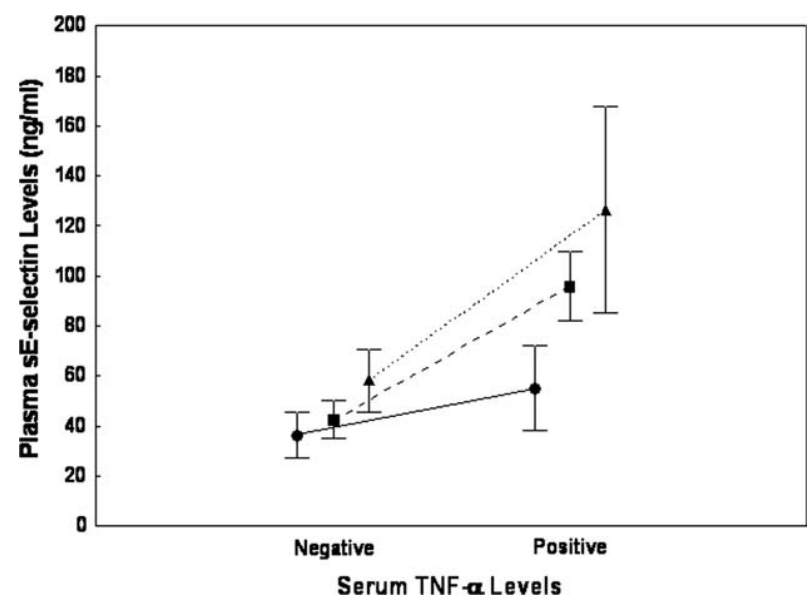

Figure 2. Two-way analysis of variance analysis of $\mathrm{sE}$-selectin levels in colorectal cancer patients stratified on the basis of carcinoembryonic antigen (CEA) mRNA positivity by reverse transcriptase-polymerase chain reaction (RT-PCR) is shown, with positive $(>20 \mathrm{pg} / \mathrm{mL})$ or negative $(\leq 20 \mathrm{pg} / \mathrm{mL})$ tumor necrosis factor- $\alpha$ (TNF- $\alpha$ ) levels. Closed circles indicate patients negative for CEA mRNA by RT-PCR; closed squares, patients with moderate CEA mRNA positivity by RT-PCR; closed triangles, patients with strong CEA mRNA positivity by RT-PCR; vertical bars, $95 \%$ confidence intervals. $F=4.68, P<.02$. negative or positive CEA mRNA expression in peripheral blood (Fig. 2). Conversely, median presurgical sE-selectin levels of patients with positive TNF- $\alpha$ levels and negative CEA mRNA expression in peripheral blood were significantly lower $(46.8 \mathrm{ng} / \mathrm{mL})$ than those observed in patients with moderate $(88.9 \mathrm{ng} / \mathrm{mL}, P<.001)$ or strong $(126.5$ $\mathrm{ng} / \mathrm{mL}, P<.001$ ) CEA mRNA positivity (Fig. 2 ).

Clinical information on postoperative follow-up was available from all primary CRC patients. Over a median follow-up period of 3 years, $49(79 \%)$ of the 62 patients remained free of disease, whereas $13(21 \%)$ patients experienced relapsing disease (stage II, $\mathrm{n}=3$; stage III, $\mathrm{n}=9$; stage IV, $\mathrm{n}=1$; median follow-up: 20 months, range, 4-103). No differences were observed in age, sex, grade, or site of primary tumor between patients with and without recurrence (data not shown). Presurgical $\mathrm{sE}$-selectin levels were above the cutoff in approximately $46 \%$ of the relapsing patients, compared with $6 \%$ (chisquare, $13.3 ; P=.0003)$ of the patients who remained free of disease. Multivariate regression analysis was then performed including recurrence as the dependent variable and age, sex, grade, site of primary tumor, stage of disease, CEA mRNA, and presurgical sE-selectin, TNF- $\alpha$, CEA, CA 19-9, and CA 72-4 tumor marker levels as the independent variables. The final model by forward stepping showed that both stage of disease (regression coefficient, $0.408 ; P=.0005)$ and elevated presurgical sE-selectin levels (regression coefficient, $0.432 ; P=.0002$ ) were independent predictors of recurrent disease (Table 2).

sE-Selectin level and CEA mRNA RT-PCR analysis during postsurgical follow-up was performed in 33 primary CRC patients. As shown in Table 3, sE-selectin levels significantly decreased during postsurgical follow-up in patients in whom positive CEA mRNA by RT-PCR

Table 2. Multivariate Regression Analysis of Disease Recurrence Rates in Colorectal Cancer Patients

\begin{tabular}{lcclc} 
Predictor Variable & \multicolumn{2}{c}{ Univariate Analysis } & \multicolumn{2}{c}{ Multivariate Analysis } \\
& $\boldsymbol{\beta}$-Coefficient (SE) & $\boldsymbol{P}$ & $\boldsymbol{\beta}$-Coefficient (SE) & $\boldsymbol{P}$ \\
Sex & $0.091(0.118)$ & .445 & Out \\
Age & $0.104(0.123)$ & .402 & Out & .0004 \\
Stage of disease & $0.320(0.144)$ & .031 & $0.408(0.109)$ & Out \\
Site of primary & $0.147(0.137)$ & .288 & Out \\
Grade & $0.026(0.142)$ & .854 & Out \\
CEA mRNA & $0.161(0.138)$ & .249 & Out \\
Serum CEA levels & $0.077(0.132)$ & .563 & Out \\
Serum CA 19-9 levels & $0.014(0.135)$ & .918 & Out \\
Serum CA 72-4 levels & $-0.076(0.135)$ & .575 & Out \\
Serum TNF- $\alpha$ levels & $0.196(0.133)$ & .146 & $0.432(0.109)$ & .0002 \\
Baseline sE-selectin levels & $0.341(0.142)$ & .021 & & \\
\hline SE indicates standard error: CEA & & &
\end{tabular}



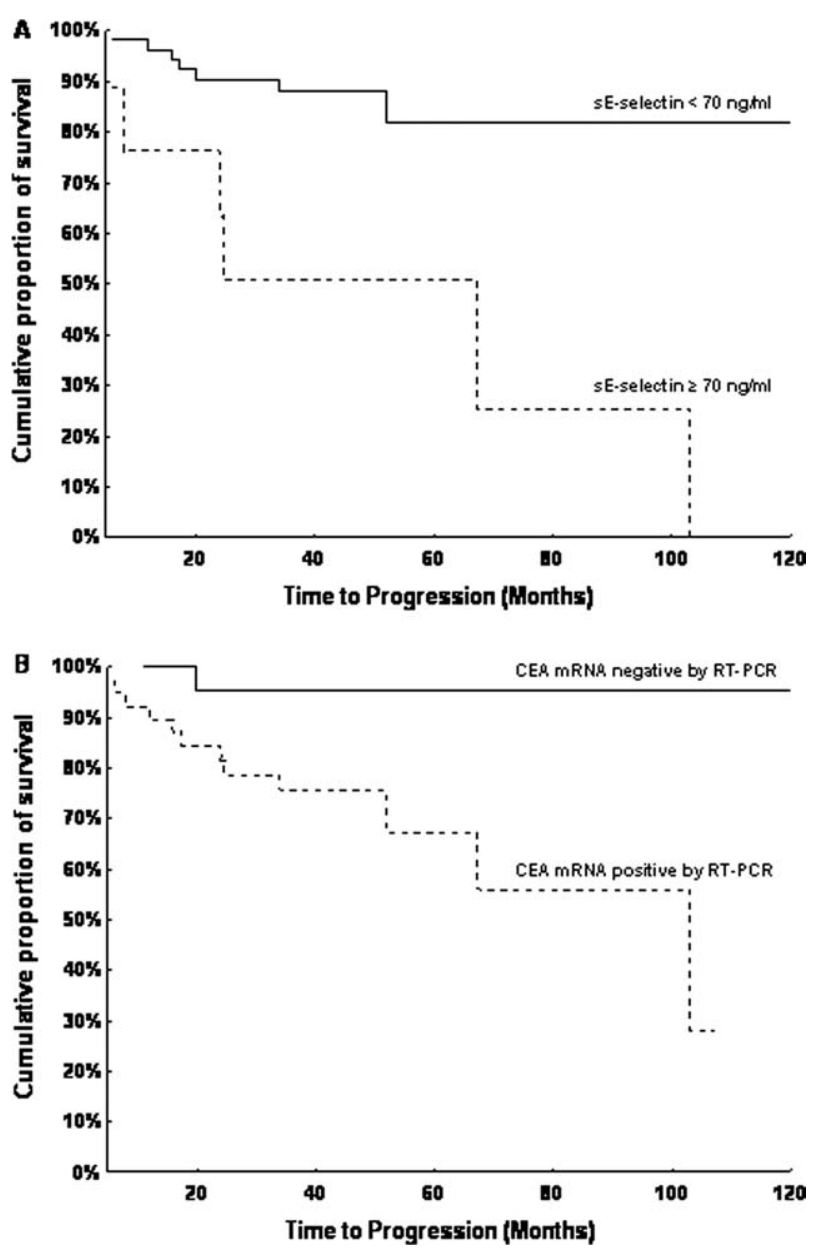

Figure 3. Kaplan-Meier analysis of recurrence-free survival time of colorectal cancer patients is stratified on the basis of (A) sE-selectin levels (Cox-Mantel test, 3.5; $P<.001$ ) or (B) carcinoembryonic antigen (CEA) mRNA positivity by reverse transcriptase-polymerase chain reaction (RT-PCR) (CoxMantel test, 2.37; $P<.02$ ).

became negative. Conversely, those patients in whom negative CEA mRNA became positive, or who had a persistent CEA mRNA positivity by RT-PCR during followup, did not show any significant change of $s$ E-selectin. No differences were observed for CEA levels (Table 3).

Figure 3A demonstrates the Kaplan-Meier recurrence-free survival curves for nonmetastatic CRC patients with low (below the cutoff value) or high (above the cutoff value) presurgical $s E$-selectin levels. As shown, high presurgical sE-selectin levels were associated with an increased recurrence rate compared with patients with low levels of this molecule in the overall CRC population (Cox-Mantel test, 3.5; $P=.0005)$. Similar results were obtained when analysis was restricted to a subgroup of stage II only patients (Cox-Mantel test, 2.6; $P=.01$ ). 


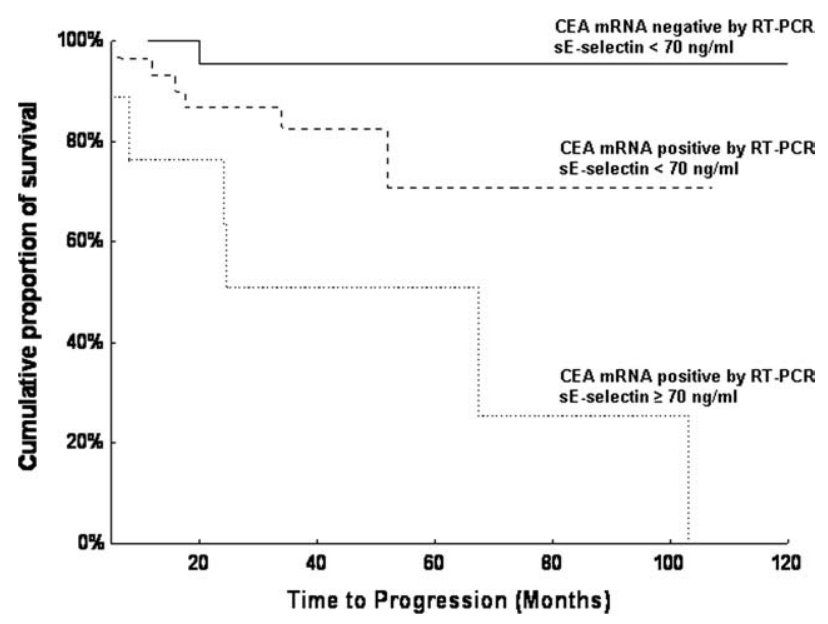

Figure 4. Kaplan-Meier analysis of recurrence-free survival time of colorectal cancer patients is stratified on the basis of positive sE-selectin levels ( $>$ the cutoff value of $70 \mathrm{ng} / \mathrm{mL}$ ) and/or carcinoembryonic antigen (CEA) mRNA positivity by reverse transcriptase-polymerase chain reaction (RT-PCR) (log-rank statistic, 8.71; $P<.02$ ).

Similarly, CEA mRNA positivity by RT-PCR was associated with an increased recurrence rate compared with negative patients (Cox-Mantel test, 2.37; $P=.018$ ) (Fig. 3B). CEA mRNA and presurgical sE-selectin were both positive or negative in 9 and 23 patients, respectively, whereas CEA mRNA positivity was associated with negative presurgical $s E$-selectin levels in the remaining 30 patients. Figure 4 shows recurrence-free survival KaplanMeier curves for patients stratified according to CEA mRNA positivity by RT-PCR and presurgical serum $s E-$ selectin levels. As shown, positivity for both CEA mRNA and $s E$-selectin before surgery had a negative prognostic impact, with a 5-year recurrence-free survival of $51 \%$; patients with positive CEA mRNA but negative presurgical sE-selectin had a 5-year recurrence-free survival rate of $71 \%$, whereas patients with negative CEA mRNA and presurgical sE-selectin had a 5-year recurrence-free survival rate of $95 \%$ (chi-square, $8.71 ; P=.013$ ).

\section{DISCUSSION}

The ability of tumor cells to secrete macrophage-activating soluble factors or activate Kupffer cells through cellcell contact has been investigated as to their role in determining the nature of the hepatic host proinflammatory response and thereby in regulating the arrest, localization, and fate of the metastasizing cells. ${ }^{3,4}$ In particular, recent experimental studies have demonstrated that CEA is capable of triggering a molecular cascade beginning with increased local production of TNF- $\alpha$ and culminating in up-regulated expression of E-selectin on the endothelium. ${ }^{6,7}$

Here we report for the first time that detection of blood-borne cells expressing CEA mRNA represent a prognostic factor for CRC recurrence, as previously suggested by other authors, ${ }^{16-18}$ but mostly in patients with elevated baseline sE-selectin levels. In fact, the 5-year survival rate of primary CRC patients with positive CEA mRNA expressing cells in the peripheral blood, but negative presurgical sE-selectin levels (71\%), was significantly higher than that of patients with both positive CEA mRNA and positive sE-selectin (51\%, Cox-Mantel test, $2.29 ; P=.02)$. This observation may partially explain the negative conclusions reached by other authors, who suggested that the presence of circulating tumor cells might be of little value as a prognostic factor, because they appeared not to be associated with conventional prognostic factors, namely the presence of metastatic disease. ${ }^{20}$ The findings here of a significant association between increased sE-selectin and positive CEA mRNA-expressing cells in the peripheral blood of patients with low-grade inflammatory status (as evidenced by increased levels of TNF- $\alpha$ ) fuel the hypothesis that circulating tumor cells may, indeed, represent a prognostic factor, but only in those patients in whom they are capable of triggering the molecular changes demonstrated in the studies cited above, ${ }^{6,7}$ and suggest that circulating tumor cells might be responsible for macrophage activation in vivo, release of proinflammatory cytokines, and up-regulation of vascular E-selectin, which can be detected in its soluble form on shedding from the endothelial cell surface. This hypothesis is also consistent with the finding that the highest $\mathrm{sE}$ selectin levels were detected in patients highly expressing CEA mRNA and with positive TNF- $\alpha$ levels compared with patients with negative levels of both variables.

Of interest, CEA mRNA positivity by RT-PCR, but not CEA protein levels, predicted the occurrence of vascular inflammation, as evidenced by increased $s E$-selectin levels, in multivariate analysis. This might be because of the occurrence of normal CEA levels in a high percentage of our patients (24\%) and its lack of association with the positivity of its mRNA in peripheral blood cells. It must be noted that, overall, we observed a correlation between serum CEA and sE-selectin levels $(\mathrm{P}=0.28, P<.05)$, as previously reported, ${ }^{8}$ but this association was completely lost at multivariate analysis. We are aware that, given the multiple pathophysiological changes known to be associated with cancer, statistical correlation does not 
necessarily indicate a causal relationship, and we cannot conclusively define the events predisposing to endothelial activation and E-selectin up-regulation. However, we might hypothesize that blood-borne CEA-expressing tumor cells, or their released products, may play a crucial role in triggering the host proinflammatory response, thus facilitating tumor cell adhesion, rolling, and diapedesis of the inflamed endothelium at metastatic sites.

Although the results reported here do not allow us to provide a definite answer on the causal relationship between blood-borne tumor cells and soluble E-selectin, the present study provides further evidence on the prognostic value of circulating tumor cells for the recurrencefree survival time of CRC patients, as previously suggested by other authors. ${ }^{16-19}$ In addition, we demonstrate for the first time that presurgical serum sE-selectin levels may also behave as a useful prognostic indicator for recurrent CRC. This finding is in agreement with previously published observations indicating that elevated sE-selectin concentration was associated with a bad prognosis for recurrencefree survival and overall survival in patients with lymph node-negative breast cancer, ${ }^{21}$ and that E-selectin could be detected on the membranes of peritumoral endothelial cells, but not on breast epithelial cells, using PCR in situ hybridization. ${ }^{22}$ Finally, the results presented here suggest that a combination of both presurgical blood-borne CEAexpressing tumor cells and sE-selectin levels might offer a better prognostic indication than either marker alone.

These hypotheses require detailed experimental evaluation before their ultimate significance can be determined. Nevertheless, we hope that our study will prompt investigators to design new studies to better understand the relationship between CEA and $s E$-selectin in disease progression, as well as their contribution as a prognostic factor for CRC. Although our study may be limited by the small sample size, the results reported here strongly suggest that detection of CEA mRNA-positive bloodborne cells in CRC patients with vascular inflammation might provide useful prognostic information in terms of recurrence-free survival, and may help in the choice of more aggressive treatment and/or more strict follow-up procedures in a subgroup of patients who are at high risk of recurrence. New prospective studies specifically designed to address this issue are warranted.

\section{CONFLICT OF INTEREST DISCLOSURES}

Partially supported by grants "Alleanza contro il Cancro" and by the Italian Ministry of Health grants RFPS-2006-7-342220 and ACC-WP 3/1b.

\section{REFERENCES}

1. Gout S, Tremblay PL, Huot J. Selectins and selectin ligands in extravasation of cancer cells and organ selectivity of metastasis. Clin Exp Metastasis. 2008;25:335-344.

2. Tremblay PL, Huot J, Auger FA. Mechanisms by which Eselectin regulates diapedesis of colon cancer cells under flow conditions. Cancer Res. 2008;68:5167-5176.

3. Khatib AM, Auguste P, Fallavollita L, et al. Characterization of the host proinflammatory response to tumor cells during the initial stages of liver metastasis. Am J Pathol. 2005;167: 749-759.

4. Auguste P, Fallavollita L, Wang N, Burnier J, Bikfalvi A, Brodt P. The host inflammatory response promotes liver metastasis by increasing tumor cell arrest and extravasation. Am J Pathol. 2007;170:1781-1792.

5. Sturm JW, Magdeburg R, Berger K, et al. Influence of TNFA on the formation of liver metastases in a syngenic mouse model. Int J Cancer. 2003;107:11-21.

6. Aarons CB, Bajenova O, Andrews C, et al. Carcinoembryonic antigen-stimulated THP-1 macrophages activate endothelial cells and increase cell-cell adhesion of colorectal cancer cells. Clin Exp Metastasis. 2007;24:201-209.

7. Thomas SN, Zhu F, Schnaar RL, Alves CS, Konstantopoulos K. Carcinoembryonic antigen and CD44 variant isoforms cooperate to mediate colon carcinoma cell adhesion to E- and L-selectin in shear flow. J Biol Chem. 2008;283: 15647-15655.

8. Roselli M, Guadagni F, Martini F, et al. Association between serum carcinoembryonic antigen and endothelial cell adhesion molecules in colorectal cancer. Oncology. 2003; 65:132-138.

9. Neumaier M, Gerhard M, Wagener C. Diagnosis of micrometastases by the amplification of tissue-specific genes. Gene. 1995;159:43-47.

10. Mori M, Mimori K, Ueo H, et al. Molecular detection of circulating solid carcinoma cells in the peripheral blood: the concept of early systemic disease. Int J Cancer. 1996;68:739-743.

11. Guadagni F, Roselli M, Cosimelli M, et al. Quantitative analysis of CEA expression in colorectal adenocarcinoma and serum: lack of correlation. Int J Cancer. 1997;72:949954.

12. Wanebo HJ, Rao B, Pinsky CM, et al. Preoperative carcinoembryonic antigen levels as a prognostic indicator of colorectal cancer. N Engl J Med. 1978;299:448-451.

13. Paterlini-Brechot P, Benali NL. Circulating tumor cells (CTC) detection: clinical impact and future directions. Cancer Lett. 2007;253:180-204.

14. Wharton RQ, Jonas SK, Glover C, et al. Increased detection of circulating tumor cells in the blood of colorectal carcinoma patients using 2 reverse transcription-PCR assays and multiple blood samples. Clin Cancer Res. 1999;5:41584163.

15. Guadagni F, Kantor J, Aloe S, et al. Detection of bloodborne cells in colorectal cancer patients by nested reverse transcription-polymerase chain reaction for carcinoembryonic antigen messenger RNA: longitudinal analyses and demonstration of its potential importance as an adjunct to multiple serum markers. Cancer Res. 2001;61:2523-2532.

16. Wang JY, Wu CH, Lu CY, et al. Molecular detection of circulating tumor cells in the peripheral blood of patients with colorectal cancer using RT-PCR: significance of the prediction of postoperative metastasis. World J Surg. 2006; 30:1007-1013. 
17. Sadahiro S, Suzuki T, Maeda Y, et al. Detection of carcinoembryonic antigen messenger RNA-expressing cells in peripheral blood 7 days after curative surgery is a novel prognostic factor in colorectal cancer. Ann Surg Oncol. 2007;14:1092-1098.

18. Wang JY, Lin SR, Wu CH, et al. Multiple molecular markers as predictors of colorectal cancer in patients with normal perioperative serum carcinoembryonic antigen levels. Clin Cancer Res. 2007;13:2406-2413.

19. Gervasoni A, Monasterio Munoz RM, et al. Molecular signature detection of circulating tumor cells using a panel of selected genes. Cancer Lett. 2008;263:267-279.
20. Sadahiro S, Suzuki T, Tokunaga N, et al. Detection of tumor cells in the portal and peripheral blood of patients with colorectal carcinoma using competitive reverse transcriptasepolymerase chain reaction. Cancer. 2001;92:1251-1258.

21. Hebbar M, Revillion F, Louchez MM, Fournier C, Bonneterre J, Peyrat JP. Prognostic value of circulating soluble Eselectin concentrations in node-negative breast cancer patients. Clin Cancer Res. 1999;5:1427-1433.

22. Shaker OG, Ay El-Deen MA, Abd El-Rahim MT, Talaat RM. Gene expression of E-selectin in tissue and its protein level in serum of breast cancer patients. Tumori. 2006;92: 524-530. 